\title{
Una Ofrenda de Cerámica Ceremonial Wari en La Oroya, Valle de Acari, Perú
}

\section{A Wari Ceremonial Ceramic Offering in La Oroya, Acari Valley, Peru}

Lidio M. VALDEZ

\section{RESUMEN}

El objetivo central de este trabajo es describir y discutir el reciente hallazgo de un depósito de cerámica ceremonial Wari, piezas que habiendo sido intencionalmente rotas fueron enterradas. El referido hallazgo proviene del sitio arqueológico de La Oroya, del valle de Acari, Perú. El hallazgo se produjo en circunstancias que los vecinos del poblado de Acari, en coordinación con la Cruz Roja, venían abriendo zanjas para instalar los sistemas de alcantarillado. Dicha excavación cortó de manera vertical una sección del depósito, exponiendo en el corte una deposición de cerámica de más de un metro de profundidad. Por cuanto el hallazgo es único y corría el peligro de ser destruido por completo, se tomó la decisión de intervenir para registrar y recuperar la deposición y de este modo evitar su definitiva destrucción.

Palabras claves: Horizonte Medio, Wari, Ofrendas de cerámica, Valle de Acarí

\section{Abstract}

The aim of this paper is to describe and discuss the recent unexpected finding of a cache of ceremonial Wari ceramics at the site of La Oroya, in the Acari Valley, on the Peruvian south coast region. The deposit was found during the excavation of trenches in the 
streets of Acari to establish the sewer system of the town. One of those trenches cut a deposit consisting of hundreds of sherds from large polychrome vessels resembling those from Conchopata, in the Ayacucho Valley. Because the finding is the first of its kind for the Acari Valley and that its destruction was inevitable, it was decided to carry out an emergency excavation in order to document and uncover the deposit.

Key words: Middle Horizon, Wari, Ceramic offerings, Acari Valley

\section{INTRODUCCIÓN}

"En la época $1 B$ del Horizonte Medio la nueva religión fue más firmemente establecida en la región de Ayacucho y Huari, y esta área constituyó el centro de un gran movimiento expansivo que llegó a la costa desde Acari hasta Chancay y en la sierra hasta Huarás..." (Menzel 1964:67).

Desde el primer hallazgo efectuado en 1927, en el sitio de Pacheco del valle de Nasca, de una inmensa cantidad de cerámica finamente elaborada que, habiendo sido intencionalmente rotas fueron posteriormente enterradas (Menzel 1964; Schreiber 1999; Cook 2001), más el posterior descubrimiento de hallazgos similares en Conchopara (Menzel 1964; Isbell 2000; Isbell \& Cook 1987, 2002; Cook 1984, 2001) y Ayapata (Ravines 1977) (Figura 1), se llegó a conocer que durante el Horizonte Medio (circa 550 - 1000 d.C.) una práctica Wari de particular importancia fue la destrucción deliberada de las mejores piezas de cerámica, o mejor dicho urnas, finamente decoradas que posteriormente habían sido enterradas. Los posteriores descubrimientos de depósitos similares nuevamente en Conchopata (Isbell 2000; Isbell \& Cook 2002; Ochatoma \& Cabrera 2001, 2002) y en el sitio de Maymi del valle de Pisco (Anders (1990) dejaron claro que el establecimiento y posterior expansión del estado Wari desde su centro de origen fueron acompañados por diversas actividades ceremoniales que incluyeron el sacrificio intencional de una serie de vasijas policromas (Cook 1984:51). Menzel (1964) reconoce a este tipo de depósitos como las "ofrendas de la tradición del Horizonte Medio".

Sin embargo, con la excepción de Conchopata, sitio este ubicado en el centro del desarrollo del estado Wari, y donde recientemente se han excavado nuevos depósitos (Ochatoma \& Cabrera 2002), la ocurrencia de tales 
hallazgos sigue siendo rara. En efecto, a lo largo del territorio que controló el estado Wari existen solamente un total de tres sitios donde se han recuperado las ofrendas de la tradición del Horizonte Medio ${ }^{1}$. Estos son: Pacheco en el valle de Nasca, Ayapata en Huancavelica y Maymi en el valle de Pisco. Las interrogantes que surgen, por lo tanto, son: ¿A qué se debe la rara ocurrencia de estos hallazgos? ¿Qué de particular tenían los sitios donde a la fecha se han encontrado talles hallazgos? ¿Fueron los asentamientos provinciales Wari establecidos en lugares particulares? Estas son algunas interrogantes que surgen en torno a la poca ocurrencia de tales hallazgos. Cualquiera haya sido el caso, a la fecha dichos hallazgos continúan siendo únicos. En la opinión de Glowacki y Malpass (2003) es posible que los lugares seleccionados por la administración Wari para establecer los nuevos asentamientos en las provincias no fueron al azar, sino cuidadosamente seleccionados.

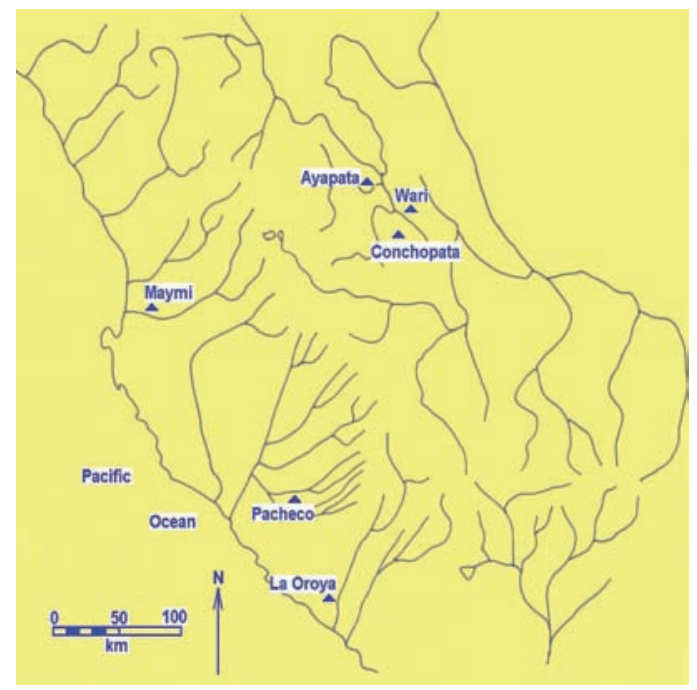

Figura 1. Ubicación del sitio arqueológico de La Oroya en relación a los otros sitios Wari con "ffrendas de la tradición del Horizonte Medio".

Figure 1. Location of the archaeological site of La Oroya in relation to other Wari sites with "offerings of the Middle Horizon tradition".

En este trabajo mi objetivo es dar a conocer el reciente hallazgo fortuito de un depósito de cerámica policroma Wari, que habiendo sido ritualmente sacrificadas habían sido enterradas siguiendo la costumbre de las ofrendas de la tradición del Horizonte Medio. Dicho hallazgo proviene del sitio arqueológico de La Oroya, ubicado en la parte media del valle de Acarí (Figura 2). El descubrimiento se hizo en circunstancias que el Municipio del mencionado poblado, en coordinación con la Cruz Roja, venía abriendo zanjas para establecer un sistema de alcantarillado. Dicha actividad resultó 
en el hallazgo de muchos restos arqueológicos, incluidos los restos humanos. El material que constituye el foco central de este trabajo había sido parcialmente expuesto, resultando en la extracción de varias piezas. Siguiendo tal descubrimiento, se vio conveniente intervenir con la finalidad de registrar el hallazgo y rescatar las piezas arqueológicas. La excavación de rescate consistió en el establecimiento de una pequeña unidad de $2 \times 2 \mathrm{~m}$, precisamente sobre el lugar donde la concentración del depósito ya había sido parcialmente expuesta.

\section{El Sitio ARQUeOlÓGICO DE LA OroYA}

En 1954 Dorothy Menzel y Francis A. Riddell llevaron adelante las primeras investigaciones arqueológicas en el valle de Acarí, de la costa sur peruana. No obstante que prestaron mayor atención al sector Inka del sitio arqueológico de Tambo Viejo, Menzel y Riddell llegaron a recorrer buena porción del valle bajo y medio, y producto de dicho esfuerzo fue la ubicación de varios sitios arqueológicos ${ }^{2}$. Entre dichos sitios, el octavo en ser registrado fue La Oroya (PV 74-8) y desde un principio identificado como un sitio perteneciente a la cultura Wari (Menzel y Riddell 1986).

La proximidad de La Oroya al actual poblado de Acarí es una de las principales razones para el estado actual de este sitio arqueológico. En efecto, lo que parece haber sido un extenso sitio arqueológico, hoy ha sido reducido a un pequeño montículo ubicado al lado Este del estadio de fútbol de Acarí, actualmente ubicado en la parte norte del poblado. El mencionado montículo ha sido a su vez severamente saqueado, quedando de este modo todo el sitio arqueológico destruido. Entretanto, en lo que fue parte del sitio se han levantado numerosas viviendas, todas actualmente ocupadas. Una pequeña plazoleta y calles completan el área urbanizada que hasta hace poco aún formaba parte del sitio arqueológico. Los propietarios de las nuevas construcciones aseguran haber recuperado numerosas piezas arqueológicas, en particular piezas de cerámica completa, durante la construcción de los nuevos inmuebles. Durante la construcción de la plazoleta también se llegó a exponer numerosos restos culturales, confirmando de este modo que el sitio arqueológico fue extenso. 


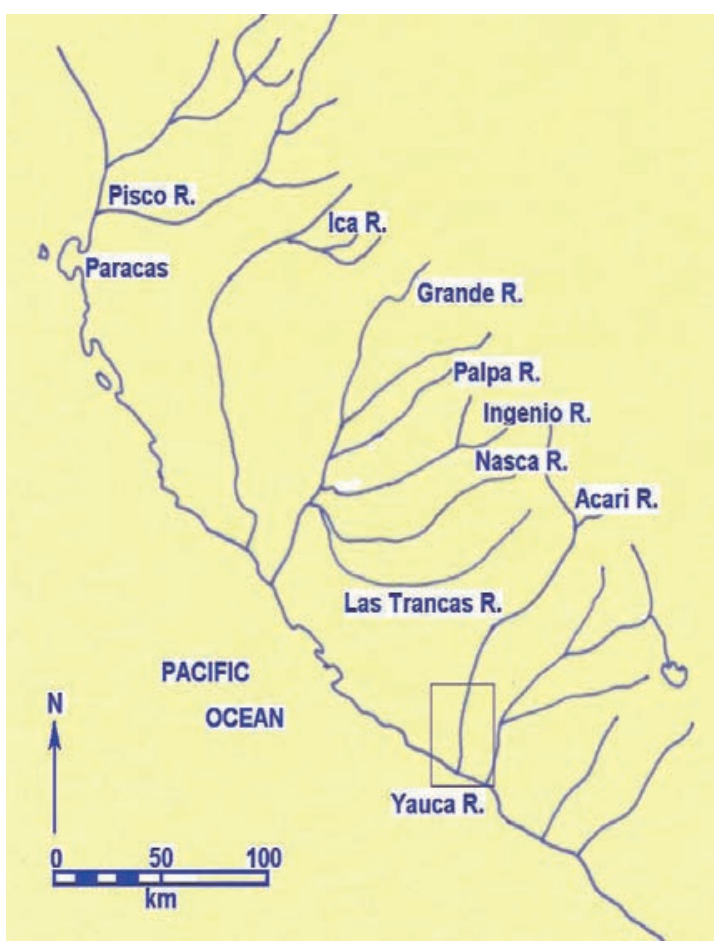

Figura 2. Ubicación de La Oroya en el valle de Acarí

Figure 2. Location of La Oroya in the Acari Valley.

Producto de esta irreparable destrucción, que en parte es producto de la carencia de una política que garantice la integridad física de los sitios arqueológicos, no existe posibilidad alguna de efectuar estudios sistemáticos en La Oroya. En consecuencia, lo aquí reportado puede que constituya una de las pocas y raras fuentes que de una u otra manera refleje lo que fue La Oroya. Sin duda alguna, con la destrucción del sitio de La Oroya se ha perdido no sólo todo un complejo arqueológico, sino también información valiosa que desafortunadamente la comunidad científica no podrá rescatar. Por este irreparable hecho, exhorto a las autoridades responsables del cuidado y protección de las ruinas arqueológicas evitar a hechos similares ${ }^{3}$.

\section{La cerámica Wari de la Oroya}

Desde el momento que se observó el depósito, con varios fragmentos ya extraídos de su contexto original, quedó evidente que éste no se trataba de un simple descubrimiento, sino de un depósito cuya ocurrencia sigue siendo rara para la arqueología Andina en general. Considerando la importancia del hallazgo, el primer paso fue inspeccionar el área adyacente al 
depósito, para luego proceder con la recuperación de los fragmentos hallados en la superficie, que como se anotó líneas atrás habían sido extraídos del depósito por los excavadores de las trincheras. Una inspección del corte producido también dejó evidente de la forma inicial del depósito, donde se pudo observar toda una superposición de fragmentos de cerámica (Figura 3). Conocedor de la particularidad del hallazgo se procedió a llevar adelante una excavación de rescate para de ese modo registrar y recuperar el contexto referido, antes de su inevitable destrucción (Valdez \& Baus 2005).

Debido a que la apertura de la zanja seguía su curso, el trabajo de rescate tenía que ser rápido para de esta manera minimizar la pérdida de pieza alguna. De este modo, el trabajo de excavación se completó en 3 días, resultando en la recuperación de un total de 37 bolsas de cerámica fragmentada. Cada bolsa pesa aproximadamente 40 kilos.

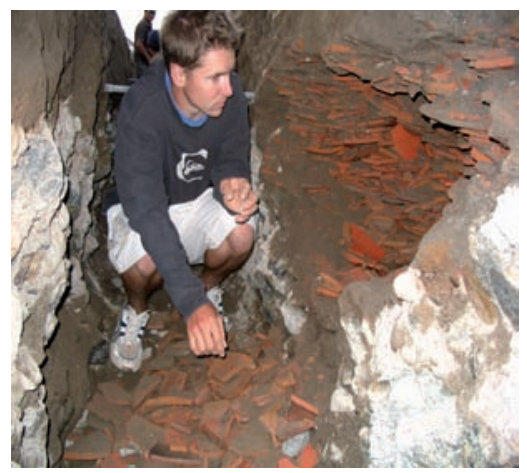

Figura 3. Vista del depósito de cerámica de La Oroya.

Figure 3. View of the ceramic deposit of La Oroya.

El hallazgo fue ubicado en la parte central de una calle que, donde una vez instaladas las tuberías del sistema de alcantarillado, se espera proceder con el asfaltado. Por lo tanto, una unidad de 2 X 2 metros de dimensión fue instalada precisamente donde la zanja había cortado parte del depósito. En seguida, se procedió a retirar una acumulación de tierra compacta que yacía sobre el depósito. Dicha formación parece haber sido el material allí depositado durante la habilitación de la calle. Por debajo de esta acumulación compacta se expuso una pequeña deposición de arena limpia y fina. Esta sólo es observable en la sección donde estaba la acumulación de los fragmentos de cerámica e indica que fue intencionalmente depositada para cubrir el depósito. Efectivamente, la arena aparece sobre los fragmentos de cerámica. Una vez retirada la arena fina, el depósito de los fragmentos de cerámica fue expuesto (Figura 4). Desde luego, la arena fina había filtrado hasta mayor profundidad. 


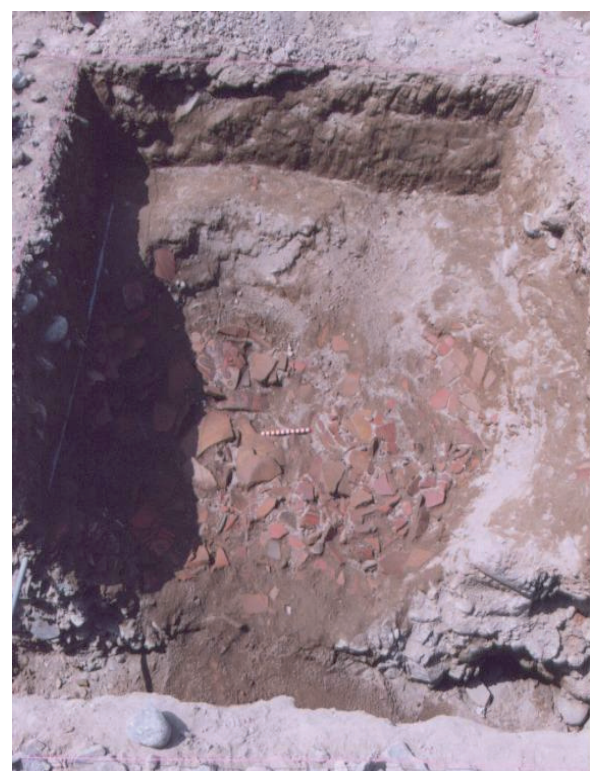

Figura 4. Excavación del depósito de cerámica.

Figure 4. Excavation of the ceramic deposit.

Los niveles inferiores de la deposición contenían claras huellas de tierra quemada, mientras los mismos fragmentos también presentan huellas de quema. La base del depósito era todavía más negra y donde los fragmentos de cerámica estaban bastante compactados. Al parecer algún producto, tal vez orgánico, fue quemado a alta temperatura y una vez enfriado llegó a solidificarse; como resultado, la excavación de la base de la deposición fue bastante dura debido a que varios fragmentos de cerámica fueron hallados pegados unos a otros. Durante el lavado, dichos fragmentos fueron remojados por varios días en bastante agua, pero sin lograr diluir el material solidificado y menos separar los fragmentos pegados.

Este hallazgo demuestra que el acto de sacrificar cerámica finamente elaborada y proceder con su posterior enterramiento no fue bastante simple. Por el contrario, la evidencia proveniente de La Oroya demuestra que previo o durante el acto de destruir las vasijas se procedió a excavar un hoyo relativamente profundo $(1.10 \mathrm{~m})$, de forma circular y de base relativamente cónica y angosta. Tal como indica la presencia de tierra quemada en la base del hoyo, una vez habilitado el hoyo se procedió a quemar algún producto orgánico, creándose de este modo una especie de fogata al interior del hoyo. Al parecer cuando el fuego estaba por consumirse, se comenzó a depositar los fragmentos de la cerámica, los mismos que fueron cuidadosamente colocados unos sobre otros y siempre en forma horizontal. Esto indica que los 
fragmentos no fueron arrojados al azar. Finalmente, una vez depositados los fragmentos se echó una acumulación de arena fina directamente sobre el depósito, sellando de este modo los fragmentos de cerámica.

La deposición en sí consiste de una acumulación de cientos de fragmentos de cerámica, generalmente gruesa. Esta sugiere que el hallazgo consiste de una ofrenda donde una serie de vasijas grandes fueron sacrificadas y enterradas. Estilísticamente, la decoración de los tiestos los vincula con la región de Ayacucho y con la cultura Wari en particular (Figura 5). Algunas muestras de las fotos tomadas de las piezas fueron posteriormente enviadas a Patricia Knobloch para su identificación estilística. De acuerdo a dicho reconocimiento, los tiestos son un derivado del estilo Chakipampa 1B (Figura 6). Knobloch también sostiene que las piezas de La Oroya guardan mucho en común con piezas recuperadas en el valle de Moquegua (ver, Owen \& Goldstein 2000: Fig. 9). Esta observación confirma la inicial apreciación de Menzel (1964), quien efectivamente sostuvo que la incorporación del valle de Acarí al control Wari tomó lugar durante el Horizonte Medio 1B.

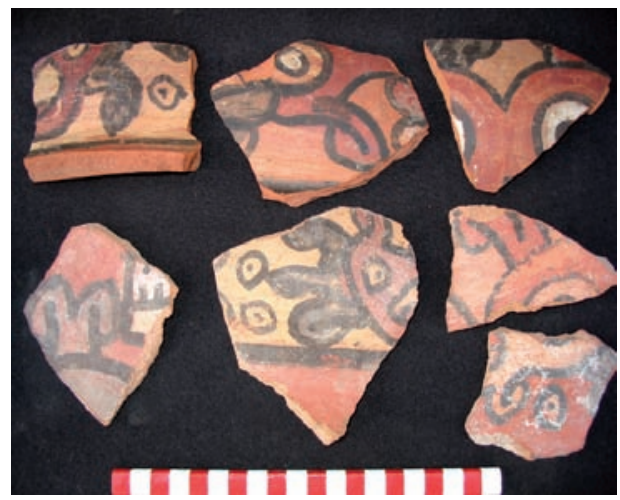

Figura 5. Cerámica del depósito de La Oroya con filiación ayacuchana. Figure 5. Ceramics from the deposit of La Oroya with highland Ayacucho origins.

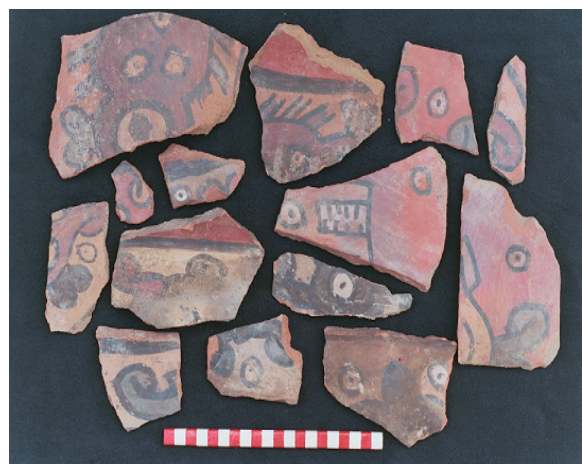

Figura 6. Cerámica del depósito de La Oroya con diseños Chakipampa $1 B$. 
Figure 6. Ceramics from the deposit of La Oroya with Chakipampa $1 B$ designs.

En cuanto a decoración se refiere, los motivos presentes en los fragmentos son los mismos o copias de aquellos que frecuentan en las vasijas Okros y Chakipampa del valle de Ayacucho, el centro de origen del Estado Wari. Sin embargo, las muestras de La Oroya no tienen la misma perfección y menos el fino acabado que las piezas encontradas en Ayacucho (Figura 7), aunque es notable el acercamiento entre ambos materiales, la misma que sugiere presencia de personal Wari dirigiendo la forma como los motivos debieron haberse ejecutado. Es decir, es posible que quienes manufacturaron y decoraron las vasijas fueron artesanos locales de Acarí, pero siguiendo las órdenes de oficiales Wari establecidos en Acarí.

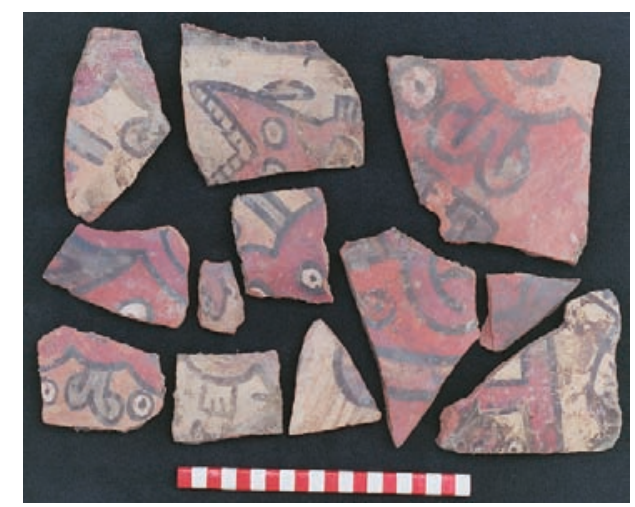

Figura 7. Cerámica decorada recuperada del depósito de La Oroya.

Figure 7. Decorated ceramics from the deposit of La Oroya.

Además de la cerámica de filiación ayacuchana, el depósito de La Oroya contenía también varias piezas de cerámica del estilo Cajamarca (Figura 8). Este estilo se caracteriza por su pasta de kaolín, superficie blanca y diseños generalmente geométricos, rellenados con espirales y motivos adicionales. Otra característica de la cerámica Cajamarca es su distinguida base de pedestal (Figura 9). Menzel anotó que la cerámica Cajamarca fue uno de los pocos estilos foráneos que mantuvo su prestigio dentro del estado Wari (Topic 1991:237), aunque su ocurrencia en la ciudad de Wari del valle de Ayacucho al parecer es más común en contextos pertenecientes al Horizonte Medio 2B (Menzel 1964:72). 


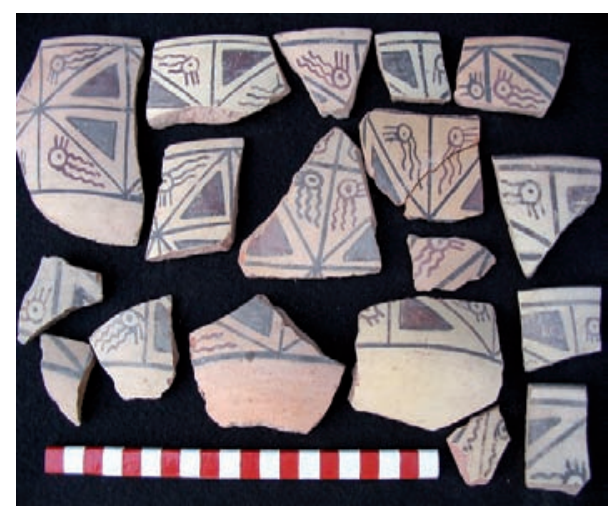

Figura 8. Cerámica del estilo Cajamarca proveniente del depósito de La Oroya.

Figure 8. Cajamarca style ceramics from the deposit of La Oroya.

Es oportuno insistir que la cerámica Cajamarca es representativa del Horizonte Medio 2 y el único sitio donde muestras Cajamarca ocurren como objetos foráneos es en Ayapata (Ravines 1977). Para esclarecer este aspecto, fotografías de la cerámica de La Oroya fueron enviadas a Dorothy Menzel. En su respuesta (comunicación personal, Noviembre 2005), Menzel señala que el depósito de La Oroya más parece representar un contexto perteneciente al Horizonte Medio 2A. Menzel menciona que la identificación de Knobloch del material de La Oroya como "imitación” de Chakipampa B podría mejor ser interpretado como "derivado" de Chakipampa B, el cual es Horizonte Medio 2A. En la opinión de Menzel, la cerámica del Horizonte Medio 1B y Horizonte Medio 2A, si bien presentan diferencias significativas, también comparten varios elementos. Menzel en ningún momento cuestiona la identificación de Knobloch, sino hace resaltar el hecho que los diseños del animal con joroba que aparecen en Horizonte Medio 1B también están presentes en Ayapata, nuevamente un contexto del Horizonte Medio 2. 


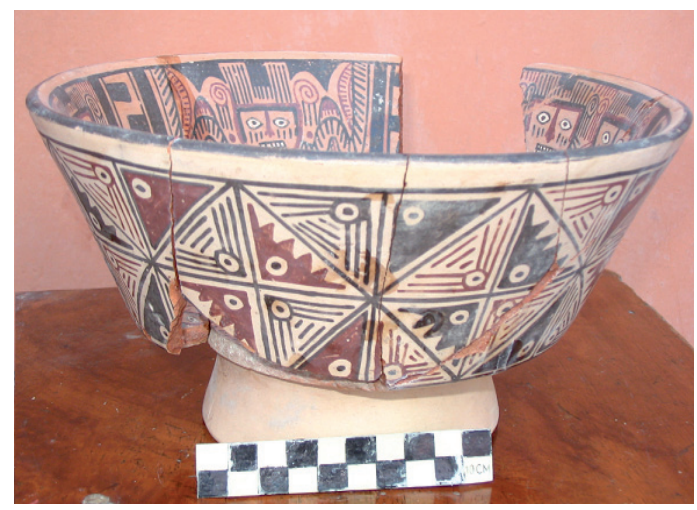

Figura 9. Vajilla en el estilo Cajamarca con su característica base pedestal proveniente del depósito de La Oroya.

Figure 9. Cajamarca style vessel with its characteristic pedestal base from the deposit of La Oroya.

\section{Ofrendas de la TRAdición DEL HORIZONTE MEdio}

Tal como se anotó líneas adelante, las ofrendas de la tradición del Horizonte Medio siguen siendo raros. En el mismo valle de Ayacucho, dichas ofrendas sólo han sido excavadas en el sitio de Conchopata (Isbell \& Cook 1987, 2002; Ochatoma \& Cabrera 2001, 2002). Por lo tanto, para el valle de Ayacucho, el sitio de Conchopata constituye un sitio excepcional en tanto que provee información valiosa con respecto a la forma como la ideología Wari se desarrolló. Al mismo tiempo, dichas evidencias anotan que Conchopata jugó un rol de mucha importancia en el proceso del establecimiento de la nueva ideología Wari, privilegio este que posteriormente cedió a la ciudad capital de Wari. La mayor presencia de hallazgos con significado ritual que datan a las fases iniciales del desarrollo del Estado Wari en Conchopata (Cook 2001), apunta precisamente hacia dicha dirección. Se entiende a su vez que los hallazgos de Conchopata anteceden a los otros hallazgos provenientes de las provincias. Por ejemplo, Menzel (1968:49) observó que la presencia Wari en el valle de Nasca (Pacheco) se dio durante el Horizonte Medio 1B.

Ahora, ¿qué fue de Ayapata, Maymi y Pacheco? El caso de Ayapata podría tal vez tener alguna relación con el hecho de que este estaba en el paso necesario hacia el valle del Mantaro, una región bastante fértil y que probablemente no tardó en captar el interés del recientemente establecido Estado Wari. El de Maymi también merece ser explorado siguiendo el mismo razonamiento, o podría ser el hecho que Maymi fue establecido cerca 
de algún centro local de particular importancia y prestigio aun por ser ubicado. Por su parte, Pacheco gana más peso si se mantiene esta perspectiva. Recordemos que Pacheco fue establecido en las proximidades de Cahuachi (Silverman 1993), un centro ceremonial pre-Wari de mucho prestigio en la costa sur durante el periodo previo a la expansión del Estado Wari. Al mismo tiempo, la presencia de las Líneas de Nasca, precisamente al norte de Pacheco, sugieren que el establecimiento de Pacheco fue estratégico. Por lo tanto, la ubicación de Pacheco parece que no fue al azar, sino fue intencional y parece que fue orientado a contrarrestar y/o absorber el prestigio de Cahuachi (Valdez 1994a).

Si esta línea de interpretación es válida, ¿cuál habría sido la razón para el establecimiento del sitio de La Oroya en la parte media del valle de Acarí? ¿Había también algún asentamiento de particular importancia en sus inmediaciones? Desafortunadamente, en Acarí no se ha identificado sitio alguno de la importancia de Cahuachi. Sin embargo, es oportuno recordar que el principal asentamiento Inka establecido en Acarí estaba ubicado a corta distancia de La Oroya (Valdez 1996). En consecuencia, ambas administraciones tal vez tuvieron las mismas razones para seleccionar esta sección del valle. Lo sobresaliente de este sector del valle es la presencia de un extenso y fértil terreno agrícola. Considerando la importancia de la agricultura para el Estado Wari, e Inka, esta es una razonable sugerencia. Si la política de la expansión Wari fue conquistar recursos y mano de obra, tal como fue para el Estado Inka, esta sugerencia parece ser una explicación válida.

En todos los casos, y no obstante que las estrategias de ocupación hayan variado de una región a otra, parece probable que en el proceso de la expansión del Estado Wari la ideología jugó un papel de mucha importancia. En efecto, el Estado Wari parece no sólo haber prestado atención a la toma de regiones específicas para de ese modo expandir sus dominios, sino parace haber prestado particular atención a la consolidación de las regiones recién incorporadas. Es ahí donde al parecer la ideología Wari jugó papel primordial. Dentro de este contexto, los depósitos deben ser parte de todo un ritual que simbolizó la incorporación de nuevas tierras y nueva población.

Los depósitos conteniendo material Wari probablemente simbolizaron no sólo la presencia Wari, sino sobre todo un compromiso de parte del Estado Wari hacia con los ocupantes de la región recientemente conquistada. Primero, la evidencia de fuego observado en el depósito de La Oroya puede ser evidencia del acto de purificación de las tierras recientemente conquistadas. Segundo, una vez purificadas y ser debidamente incorporadas al dominio Wari, el Estado Wari se vio obligado a hacer entrega de una serie de 
ofrendas, o pagapus. Sólo así, al parecer, se habría cumplido con la incorporación de nuevas regiones.

El material proveniente de La Oroya ayuda poco para abarcar más esta línea de razonamiento. Sin embargo, si se considera el material hallado en Maymi (Anders 1990), en particular su iconografía, resaltan de inmediato los diseños donde sobresalen una variedad de cultivos alto-andinos. Lo mismo se podría decir acerca del material proveniente de Pacheco, donde también es notable el obvio énfasis en hacer resaltar los cultivos alto-andinos. Considerando el origen del Estado Wari, la presencia de una rica iconografía que hace resaltar a una variedad de plantas y productos, parece haber sido intencional y estratégica, pues el concepto que acompañó a dichas representaciones no pudo haber sido otro que fertilidad y prosperidad agrícola. Para una región seca y generalmente árida como la costa sur, representaciones que denotaban fertilidad y prosperidad agrícola seguramente fueron bienvenidos.

\section{La Oroya en SU CONTEXTO LOCAL}

Estudios recientes en el valle de Nasca han demostrado la escasa presencia de asentamientos Wari pertenecientes a la época $1 \mathrm{~B}$ del Horizonte Medio. Hasta hace poco, el único sitio, desafortunadamente ya destruido, fue Pacheco. Las prospecciones de Schreiber (1999:169) sólo llegaron a ubicar un pequeńo establecimiento Wari (Pataraya) ubicado en la parte alta del valle. En la opinión de Schreiber, al tiempo de la llegada Wari al valle de Nasca, este se hallaba parcialmente despoblado como resultado de un posible movimiento poblacional hacia Huaca del Loro.

El valle inmediatamente al sur de la cuenca del Río Grande de Nasca, viene a ser Acarí. A diferencia de la región de Nasca, donde se han efectuado numerosos estudios arqueológicos, Acarí permanece en la retaguardia. Como resultado, el desarrollo cultural de este valle sigue siendo incierto. Sin embargo, y al igual que en la cuenca del Río Grande de Nasca, al momento de la llegada Wari hacia el valle de Acarí había un estilo local que tiene algunos aspectos comunes con el estilo Loro de la Cuenca del Río Grande. Dicho estilo ha sido distinguido por Kent y Kowta (1994) y se caracteriza por unos vasos altos que representan como diseño principal caras antropomorfas de ojos alargados y nariz modelada. Los sitios donde ocurre dicho estilo incluyen a Tambo Viejo, Gentilar, Pellejo Chico, Chavińa y La Oroya. De todos, el más extenso parece haber sido Chavińa, sitio este ubicado cerca de la desembocadura del río Acarí (Valdez 1994b:358). Para distinguir a esta 
traidición local de Acarí del estilo Loro, prefiero identificarlo como el estilo Chavińa.

El estilo Chavińa aparece en la superficie de La Oroya, denotando que con anterioridad a la llegada Wari el lugar fue ocupado. Por lo tanto, existe la posibilidad de que al momento de la conquista Wari del valle de Acarí, La Oroya constituía un importante asentamiento, tal vez como resultado de su proximidad a la sección más fértil del valle. Cualquiera haya sido el escenario, la evidencia de que se dispone indica que en el valle de Acarí, el Estado Wari se estableció sobre un lugar que estaba ocupado. Considerando la importancia agrícola de esta sección del valle

Resumiendo, el reciente hallazgo proveniente de La Oroya permite incluir a dicho sitio en un grupo selecto de sitios Wari con ofrendas de la tradición del Horizonte Medio. Si bien para ampliar la discusión generada en este reporte se hace necesario llevar adelante los respectivos análisis, la nueva evidencia permite considerar varias posibilidades con respecto a la presencia de tales depósitos. Al mismo tiempo, la nueva evidencia permite conocer aspectos que hace poco simplemente eran desconocidos, siendo el caso particular de la presencia del fuego en dichos rituales. En la medida que nuevos hallazgos sean detectados y nuevas evidencias sean acumuladas es posible que se logre visualizar mejor este importante aspecto de la ideología Wari.

Agradecimientos: Carlos Morán, Maribel Quiroga, María Quiroga, Angus Danielson, Heather Battles, María Quiroga, Sylvere Valentin, Annalisa Christie, Patrick Kuse, Annette Baus y Regina McGowan participaron activamente en las tareas de rescate. Rosa Mazuelo, Angel Iglesias y Marko de la Cadena prestaron su apoyo incondicional durante mi estadía en Acarí. Finalmente, hago extensivo mi reconocimiento a Patricia Knobloch y Dorothy Menzel por sus apreciaciones, observaciones y comentarios con respecto a la ubicación temporal de las muestras de cerámica de La Oroya.

\section{Notas}

${ }^{1}$ Glowacki (2002:268) menciona que depósitos similares existen también en la región del Cusco, pero no menciona sitio alguno.

2 Dorothy Menzel y Francis A. Riddell realizaron sus investigaciones en Tambo Viejo formando parte del proyecto "Caminos del Inca” dirigido por Víctor von Hagen (ver Rowe 1956).

3 En particular hago alerta a la posible destrucción del sitio de Tambo Viejo ubicado al lado Sur del poblado de Acarí. Secciones de Tambo Viejo ya vienen siendo lotizadas, mientras en una buena parte del sitio ya se han levantado numerosas viviendas. De no frenar esta acción, Tambo Viejo podría correr la misma suerte que La Oroya, posibilidad que se tiene y debe de evitar. 


\section{Bibliografía}

Anders, M. 1990. "Maymi: un sitio del Horizonte Medio en el valle de Pisco". Gaceta Arqueológica Andina 17:27-39.

Cook, A. 1984. "The Middle Horizon ceramic offerings from Conchopata". Nawpa Pacha 22-23:49-90.

Cook, A. 2001. "Huari D-shaped structures, sacrificial offerings, and divine rulership". En Ritual Sacrifice in Ancient Peru, editado por E. Benson y A. Cook, pp: 137-163. University of Texas Press, Austin.

Glowacki, M. 2002. “The Huaro Archaeological Site Complex: rethinking the Huari occupation of Cuzco". En Andean Archaeology I: Variations in Sociopolitical Organization, editado por W. H. Isbell y H. Silverman, pp: 267-285. Kluwer Academic / Plenum Publishers, New York.

Glowacki, M. y M. Malpass. 2003. "Water, huacas, and ancestor worship: traces of a sacred Wari landscape". Latin American Antiquity 14 (4): 431-448.

Kent, J. y M. Kowta. 1994. "The cemetery at Tambo Viejo, Acari Valley, Peru”. Andean Past 4:109-140.

Isbell, W. H. 2000. "Repensando el Horizonte Medio". Boletín de Arqueología PUCP 4:9-68.

Isbell, W. y A. Cook. 1987. "Ideological origins of an Andean conquest state”. Archaeology 40 (4):26-33.

Isbell, W. y A. Cook. 2002. "New perspectives on Conchopata and the Andean Middle Horizon". En Andean Archaeology II: Art, Landscape, and Society, editado por H. Silverman y W. H. Isbell, pp: 249-305. Kluwer Academic / Plenum Publishers, New York.

Menzel, D. 1964. "Style and time in Middle Horizon". Nawpa Pacha 2:1106.

Menzel, D. y F. Riddell. 1986. Archaeological Investigations at Tambo Viejo, Acari Valley, Peru 1954. California Institute for Peruvian Studies, Sacramento.

Ochatoma, J. y M. Cabrera. 2001. "Arquitectura y áreas de actividad en Conchopata". Boletín de Arqueología PUCP 4:449-488.

Ochatoma, J. y M. Cabrera. 2002. "Religious ideology and military organization in the iconography of a D-shaped ceremonial precinct at Conchopata". En Andean Archaeology II: Art, Landscape, and Society, 
editado por H. Silverman y W. H. Isbell, pp: 225-247. Kluwer Academic / Plenum Publishers, New York.

Owen, B. y P. Goldstein. 2000 "Tiwanaku en Moquegua: interacciones regionales y colapso”. Boletín de Arqueología PUCP 5: 169-188.

Ravines, R. 1977. "Excavaciones en Ayapata, Huancavelica, Perú". Nawpa Pacha 15:19-45.

Rowe, J. 1956. "Archaeological explorations in southern Peru, 1954 1955”. American Antiquity 22 (2):135-151.

Schreiber, K. 1999. "Regional approaches to the study of prehistoric empires: examples fom Ayacucho and Nasca, Peru". En Settlement Pattern Studies in the Americas: Fifty Years since Virú, editado por B. Billman y G. Feinman, pp: 160-171. Smithsonian Institution Press, Washington, D.C.

Silverman, H. 1993. Cahuachi in the Ancient Nasca World. Iowa University Press, Iowa City.

Topic, T. 1991. "The Middle Horizon in northern Peru". En Huari Administrative Structure: Prehistoric Monumental Architecture and State Government, editado por W. H. Isbell y F. McEwan, pp: 233246. Dumbarton Oaks, Washington, D.C.

Valdez, L. 1994a. "New evidence for an early Nasca ceremonial role". Current Anthropology 35:675-679.

Valdez, L. 1994b. "Investigaciones arqueológicas en Ventilar, Acarî". Boletín de Lima 91-96:351-361.

Valdez, L. 1996. "Los depósitos Inka de Tambo Viejo, Acarí". Tawantinsuyo 2:37-43.

Valdez, L. y A. Baus. 2005. "Wari ceremonial ceramics from La Oroya, Acari Valley, Peru". Ponencia presentada al 24th Annual Meeting of the Northeast Conference on Andean Archaeology and Ethnohistory, Octubre 2005. American University \& Catholic University of America, Washington, D.C. 